\title{
PARAMETRIC OPTIMIZATION OF MAGNETO-RHEOLOGICAL FLUID DAMPER USING PARTICLE SWARM OPTIMIZATION
}

\author{
M.R. Azraai ${ }^{1 *}$, G. Priyandoko², A.R. Yusoff ${ }^{1}$ and M.F.F.A. Rashid ${ }^{2}$ \\ ${ }^{1}$ Faculty of Manufacturing Engineering, Universiti Malaysia Pahang, \\ 26600 Pekan, Pahang, Malaysia \\ Email: azraai@ump.edu.my \\ Phone: +609424 5800; Fax: +609424 5888 \\ ${ }^{2}$ Faculty of Mechanical Engineering, Universiti Malaysia Pahang, \\ 26600 Pekan, Pahang, Malaysia
}

\begin{abstract}
This paper presents a parametric modeling of a magneto-rheological (MR) damper using a Particle Swarm Optimization (PSO) method. The objective of this paper is to optimize the parameter values of the MR fluid damper behavior using the Bouc-Wen model. The parametric identification was imposed beforehand in replicating the behavior of the MR fluid damper. The algebraic function from a number of hysteresis models was steered by comparing selected models: Bingham, Bouc-Wen and BoucWen by Kwok. A simulation method was operated in investigating these models by employing MATLAB reliant from the model intricacy. The experimental data was presented in terms of the time histories of the displacement, the velocity and the force parameters, measured for both constant and variable current settings and at a selected frequency applied to the damper. The model parameters were determined using a set of experimental measurements corresponding to different current constant values. It has been shown that the MR damper model's response via the proposed approach is in good agreement with the MR damper test rig counterpart.
\end{abstract}

Keywords: Magneto-rheological; absorber; hysteresis; PSO.

\section{INTRODUCTION}

The application of semi-active suspension throughout the decades has shown promising potential, predominantly based on its stability and robust nature for controlling exerted vibrations, particularly in automotives [1]. Hence the important role of the damper in influencing vibration. Magneto-rheological (MR) fluid is reported to be a smart material that is able to alter its resistivity with pertinent operation. In spite of the adaptability of the MR fluid damper, the complexity of modeling its behavior has been a subject of scrutiny ever since. The MR fluid damper is a semi-active control device that alters its viscosity once magnetized $[2,3]$. The MR fluid consists of oil and a substantial amount of iron particles. The MR damper structure is shown in Figure 1. The MR fluid is lodged in the damper cylinder which allows it to flow through the orifices. Magnetisable particles submerged in the fluid actuate the MR damper. This is due to the magnetic field supplied from the magnetic choke prior to current supply, which as a consequence aligns the particles in chain-like structures perpendicular to the fluid flow [4]. Subsequently, the physical characteristics of the MR fluid are determined by the input current which controls the viscosity as the fluid changes to semi-solid form [5]. 


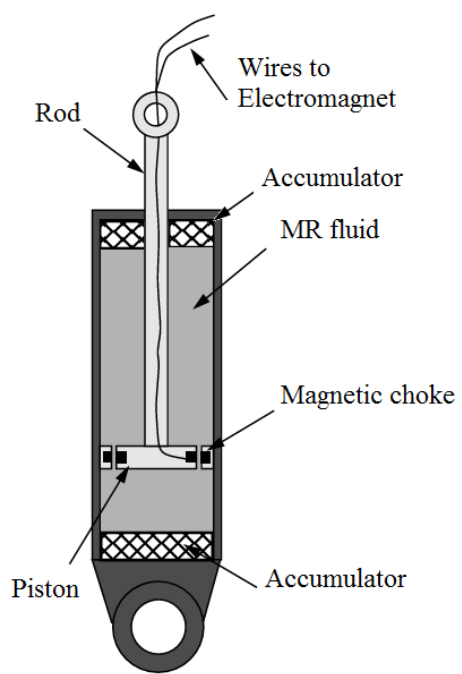

Figure 1. MR damper schematic.

Effective control of an MR damper mainly depends on understanding its nonlinear hysteretic behavior under an applied magnetic field. Therefore, the development of the distinct features and consider the non-linear behavior of the MR damper mechanism, this damper behavior was the extension of previous work [6]. The existing models can be categorized into two separate parametric and non-parametric groups. Non-parametric models are able to model the MR damper behavior in such a way that the model parameters do not illustrate the physical connections of the body [7]. Modeling an MR fluid damper which consists of various non-linear properties is a complicated phenomenon associated with a hysteresis system. A non-linear system can be characterized by implementing a memory feature. Both the instantaneous input and its preceding data contribute to the output value for a specific given time. The enactment of a hysteresis system does not respond proportionately but instead differs from the forces applied. This component offers high flexibility in a wide range of engineering applications. Both mathematical and non-mathematical approaches have been seen in various MR damper modeling publications $[8,9]$. These can be listed in two separate categories for modeling the dynamic behavior of MR dampers through either parametric or non-parametric identification techniques. Parametric techniques represent the mechanical scheme by an arrangement of physical qualities and its elements of a spring and viscous dashpot [10]. The Bouc-Wen model is an example of a parametric identification technique. It is done by curve fitting of experimental results from optimizing the parameters from the Bouc-Wen model as a semi-empirical relationship. On the other hand, the primary relationship of the input-output system model is not conjectured in a non-parametric identification technique. From a given arbitrary input, the succeeding prediction of the systems is enabled, subsequently identifying the prominent values of the input/output data to be stored. Parametric techniques are based on mechanical principles including interpretation by arranging springs and dashpots. Non-parametric techniques are used for direct dynamic modeling prediction of the output for given inputs. Although the non-parametric models can efficiently depict the MR damper behavior, the complexity in validation is taxing, with massive amounts of data required for validation. Table 1 classifies the identification methods with sub-division of the parametric models. 
Table 1. Parametric identification classification.

\begin{tabular}{|c|c|c|}
\hline \multicolumn{2}{|c|}{ Parametric } & \multirow[t]{2}{*}{ Non-parametric } \\
\hline Evolutionary model & Algebraic model & \\
\hline General Bouc-Wen (BW) & Bingham & Chebyshev polynomials \\
\hline Modified Bouc-Wen & Bouc-Wen (Kwok) & Neural networks \\
\hline $\begin{array}{l}\text { Voltage-dependent modified } \\
\text { Bouc-Wen }\end{array}$ & $\begin{array}{l}\text { Modified algebraic } \\
\text { model (Guo and } \mathrm{Hu} \text { ) }\end{array}$ & Neuro-fuzzy \\
\hline Modified Dahl model & - & - \\
\hline $\begin{array}{l}\text { Modified Lugre Friction } \\
\text { model }\end{array}$ & - & - \\
\hline
\end{tabular}

In furtherance of imitating the MR fluid damper, the Particle Swarm Optimization (PSO) method was introduced to enhance the parameter search for identification. The concept of PSO emulates the social behavior of wildlife interaction primarily in a clustered movement, for instance in a flock of birds or swarm of ants. The collaboration between Kennedy and Eberhart in [11] led to this renowned optimization method which has been seen in diverse applications ever since [12]. Subsequent to the motion of these groups, the social behavior analogy is used to acquire the best parameter value; for instance, a flock of birds looking for a source of food in a randomized formation until one bird locates food at a position which is then predicted as an optimized position. This analogy is then applied to the hysteresis model to locate the best possible value in imitation of the MR fluid damper characteristics. Identification techniques are used to model the MR fluid damper to replicate its practical behavior and are categorized as either parametric or non-parametric. The objective of this paper is parametric modeling of the MR fluid damper using the BoucWen hysteresis model proposed by Kwok [13], which is capable of being optimized by using the advocated PSO algorithm.

\section{EXPERIMENTAL SETUP}

\section{Design of Experiment}

An original shock absorber equipment was employed for orientation in developing the MR damper model. A Proton Waja shock absorber was selected as a reference due to its specifications and the unsophisticated operation sets for its assembly. However, installation of the MR fluid damper model in the Proton Waja model will introduce constraints in terms of the design, as the factors of parameters, for instance the shock absorber tube's diameter and stroke length, are already in place. Figure 1 provides an illustration of the absorber in detail. A conventional damper mainly consists of a piston rod, bearing and seal, but an MR damper comprises several additional elements, essentially an accumulator and electromagnet. Nonetheless, an MR fluid of hydrocarbon-based MRF-122EG was employed in the damper cylinder with the purpose of implying an electromagnetic behavior on the damper. The MR fluid damper is shown in Figure 2. 


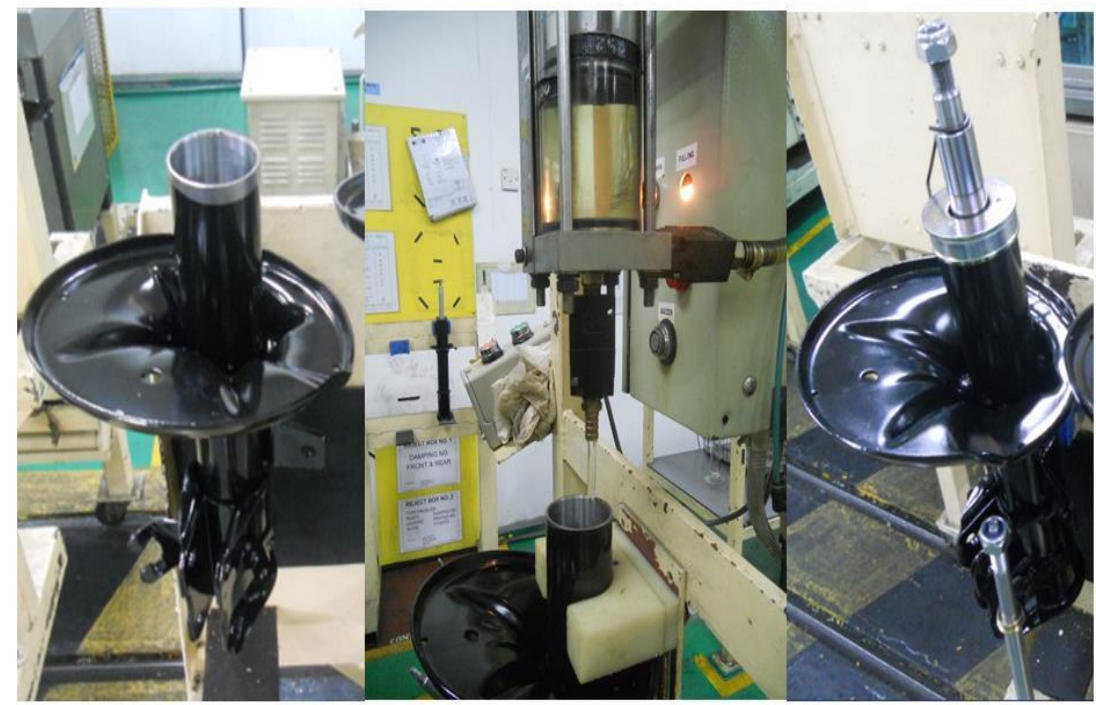

Figure 2. MR fluid damper assembly.

As for the performance of the damper, Figure 3 below illustrates the force against velocity for the original equipment. The performance of this damper is used as a point of reference in designing the MR damper. This was tested using a material testing system (MTS) machine. An evaluation method was exploited to gauge the differences between the experimental and simulated data. A basic scheme for the justifying the measurement is illustrated in Figure 4 below. Firstly, the input data (collected from the experiment) is submitted into a Synthesize program for evaluation with the programming code.

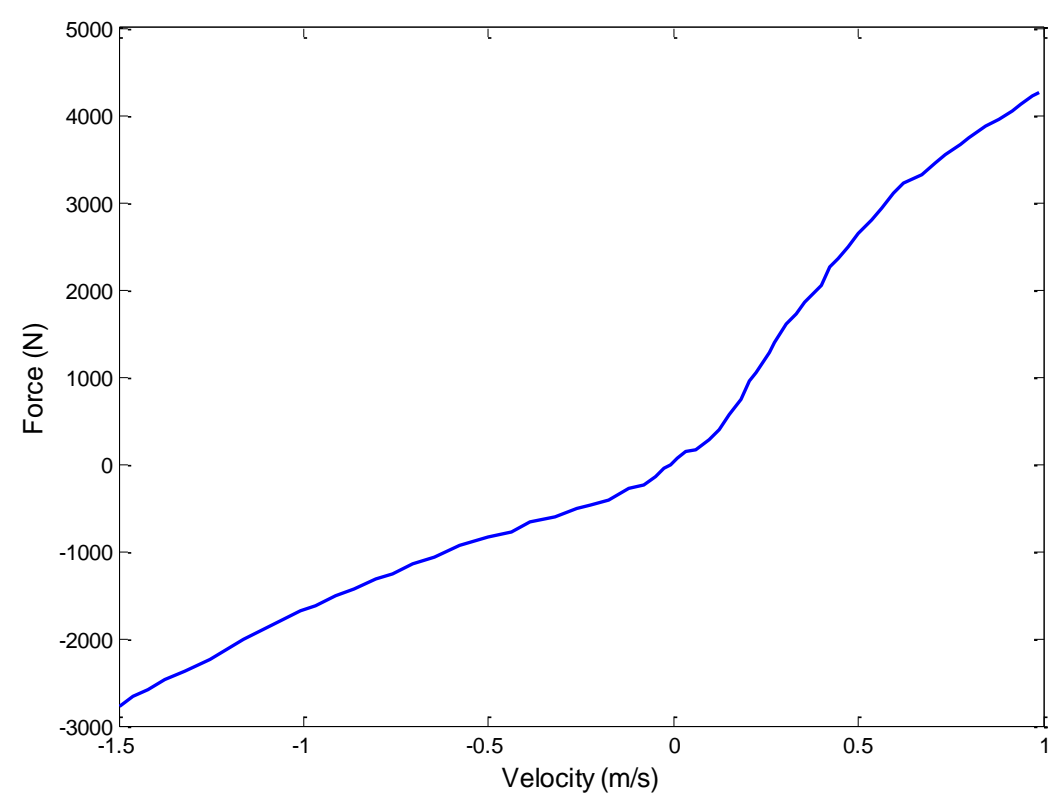

Figure 3. Force-velocity plot of original Proton Waja absorber. 


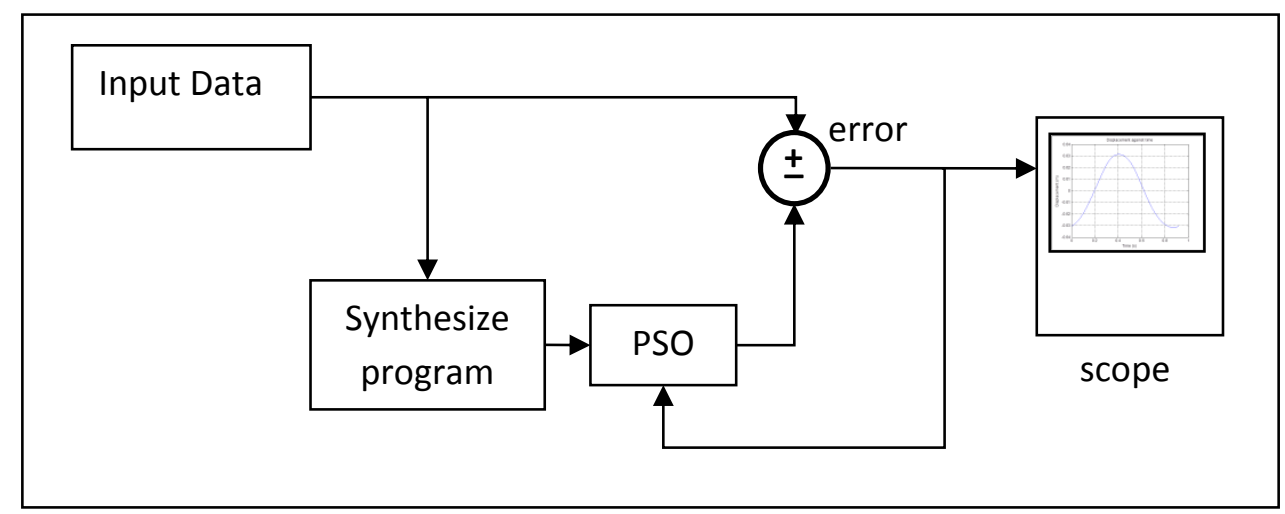

Figure 4. Error comparison gauge measurement.

The hysteresis models were incorporated into the synthesize program beforehand in order to clarify significant parameters that required optimization prior to disparity investigation with the experimental data. After altering the relevant parameters, PSO was applied to enhance the performance of the simulated hysteresis graph and compare it to the input data. Then, an evaluation was made to ensure that the error between the input data and the resultant optimized result was satisfactory. The performance of the proposed Bouc-Wen model modified by Kwok was justified by comparing it to the Bingham and original Bouc-Wen models.

\section{MATHEMATICAL MODELING}

The model proposed by Kwok uses the hyperbolic tangent function to embody the mechanical character of viscosity and stiffness corresponding to hysteresis and linear functions. The equations used are as below [13]:

$$
\begin{gathered}
F=c \dot{x}+k x+\alpha z+f_{0} \\
z=\tanh [\beta \dot{x}+\delta \operatorname{sgn}(x)]
\end{gathered}
$$

where $z$ is identical to the other model's operation except for the introduction of a hyperbolic tangent function, and the offset of the damper is the parameter $f_{0} . c, k, \alpha, \delta$, $\beta$, and $\gamma$ are model parameters to be identified, $f$ is the damping force, $c$ and $k$ are the viscosity and stiffness coefficient respectively.

Kwok [13] claims that the model offers efficiency in computational execution for parameter identification, such that carrying out the controller design is placid from the hyperbolic tangent function.

\section{PARTICLE SWARM OPTIMIZATION}

The concept of particle swarm optimization (PSO) was originally introduced by Kennedy and Eberhart in 1995 [11] as a technique for individual improvement through population cooperation and competition, which is based on the simulation of a simplified social model, such as bird flocking, fish schooling and the swarm theory. Its mechanism enhances and adapts to the global and local exploration. Some of the key advantages are that this method does not require the calculation of derivatives, that the knowledge of good solutions is retained by all particles, and that these particles in the 
swarm share information between them. PSO is less sensitive to the nature of the objective function, can be used for stochastic objective functions and can easily escape from local minima. Concerning its implementation, PSO can easily be programmed, has few parameters to regulate, and the assessment of the optimum is independent of the initial solution. Nowadays, PSO has gained much attention and wide application in various fields. The basic PSO algorithm consists of three steps, namely, generating particles' positions and velocities, velocity update, and position update. Here, a particle refers to a point in the design space that changes its position from one move (iteration) to another based on velocity updates. First, the positions, $x_{k}^{i}$, and velocities, $v_{k}^{i}$, of the initial swarm of particles are randomly generated using upper and lower bounds on the design variables values, $x_{\min }$ and $x_{\max }$, as expressed in Eqs. (.3) and (4).

$$
\begin{array}{r}
x_{0}^{i}=x_{\text {min }}+\operatorname{rand}\left(x_{\text {max }}-x_{\text {min }}\right) \\
v_{0}^{i}=\frac{\text { position }}{\text { time }}=\frac{x_{\text {min }}+\operatorname{rand}\left(x_{\max }-x_{\min }\right)}{\Delta t}
\end{array}
$$

A uniformly distributed random variable, rand, is valued from 0 to 1 . The vector format is translated to describe the velocity and position denoted by the $i$ th particle at given time $k$. The best global value, $p_{k}^{g}$, is determined from the fitness function value of a particle in the running swarm. Hence, the best position pbest is selected from each particle and correlated with all previous groups for the best global gbest. In Eq. (5), a summation mode is applied to reposition the direction of the combining swarm's influence, the particles' memory and current motion. Consequently, Eq. (.6) explains the velocity vector which is applied to update the position of the particle.

$$
\begin{gathered}
v_{k+1}^{i}=w v_{k}^{i}+c_{1} \text { rand } \frac{\left(p^{i}-x_{k}^{i}\right)}{\Delta t}+c_{2} \text { rand } \frac{\left(p_{k}^{g}-x_{k}^{i}\right)}{\Delta t} \\
x_{k+1}^{i}=x_{k}^{i}+v_{k+1}^{i} \Delta t
\end{gathered}
$$

\section{RESULTS AND DISCUSSION}

The MR fluid damper was developed and employed on the test rig. Hardware integration was successfully done and extracted to DAQ and saved as experimental results. The conditions of the experimental test were evaluated under a set of assessments as measured earlier. Then, a comparison with a noteworthy hysteresis model was performed to obtain and justify the selected model for this research. An elementary statistical analysis is presented to unveil the resolution for the nominated models. Once the selection is fulfilled, the various test conditions for the selected model are illustrated, comparing the experimental and simulated data. Next, a thorough analysis of the marginal and percentile error was conducted to enhance the declaration of the best optimized hysteresis model. The parameter values are examined to extend the understanding of significant changes that lead to formation of the curve. Table 2 presents the respective findings, with the test conditions input. 
Table 2. Mean of parametric models force error $(\mathrm{N})$.

\begin{tabular}{cccc}
\hline Test condition & \multicolumn{3}{c}{ Parametric model error (N) } \\
\cline { 2 - 4 } Current input (A) & Bingham & Bouc-Wen & Bouc-Wen/Kwok \\
\hline 0.0 & 35.917 & 20.094 & 5.452 \\
0.5 & 87.068 & 30.752 & 20.601 \\
1.0 & 110.347 & 75.829 & 18.142 \\
1.5 & 139.181 & 119.175 & 23.013 \\
2.0 & 182.453 & 150.557 & 33.201 \\
\hline Mean average & 110.993 & 79.281 & 20.082 \\
\hline
\end{tabular}

Trends of rising force error are observed for all models as the current input ascends. The largest increment is clearly distinguished from the Bingham model in the range of $30 \mathrm{~N}$ to $50 \mathrm{~N}$ as the test input extends. With a mean average of $111 \mathrm{~N}$, the Bingham model was found to be an unsuitable form for replicating the MR fluid damper behavior. The Bouc-Wen model, on the other hand, demonstrates slight improvements in the marginal error, with impartial results of $79.3 \mathrm{~N}$. Nonetheless, this irregular outcome is still inconsistent in parameter identification. Ultimately, the Bouc-Wen model by Kwok [13] presents an astounding inference on MR fluid damper performance. The divergences between the test conditions were minimal, with less than $15 \mathrm{~N}$ and a significant mean average of $20.1 \mathrm{~N}$ marginal error. The initial step in the experiments is to apply a current input of $0.0 \mathrm{~A}$. The behavior is comparable to a passive damper due to zero current exerted, so the MR fluid acts as a typical fluid damper, excluding the resistive force by unmagnetized particles. Figure 5 represents the findings using the Bouc-Wen model, making it possible to scrutinize how far the simulation data achieves the measured value. The respective figures are assigned in arrays as shown in Figure 5. From Figure 5(b) and (c), it can be judged to what extent the data relates to the corresponding hysteresis model. To support the findings, the marginal error in terms of force is shown in Figure 5(d). In this manner, inspection of the findings is performed in parallel for a straightforward verdict. Taken as a whole, the response evidenced from the test condition at $0.0 \mathrm{~A}$ presents credible results and is indicative of a decisive model for parametric identification.

In general, all of the results from the test conditions forecast by using the BoucWen model by Kwok were satisfactory, despite having a minor setback that was insignificant with respect to the MR fluid damper characteristics. As an alternative, supplementary analysis was undertaken to commit to a final verdict on verifying the parametric model as a substitute for modeling the MR fluid damper. The consequence of these results was mainly founded on the parameter values, and the indices are list in Table 3. It is shown that the comparison of existing parametric models using the proposed Bouc-Wen model modified by Kwok et al. [13] and performed by computer simulation. The selected model is optimized by imposing the PSO algorithm in order to assign the significant parameters into the best fitting model with respect to the MR fluid damper. The analysis reveals that the average percentage error for the simulation to depict the MR fluid damper's behavior is approximately $6.0 \%$ to $8.3 \%$ marginal error. This estimation is presumed to be satisfactory, recognizing the fact that several constraints were unavoidable during the process of this research. 

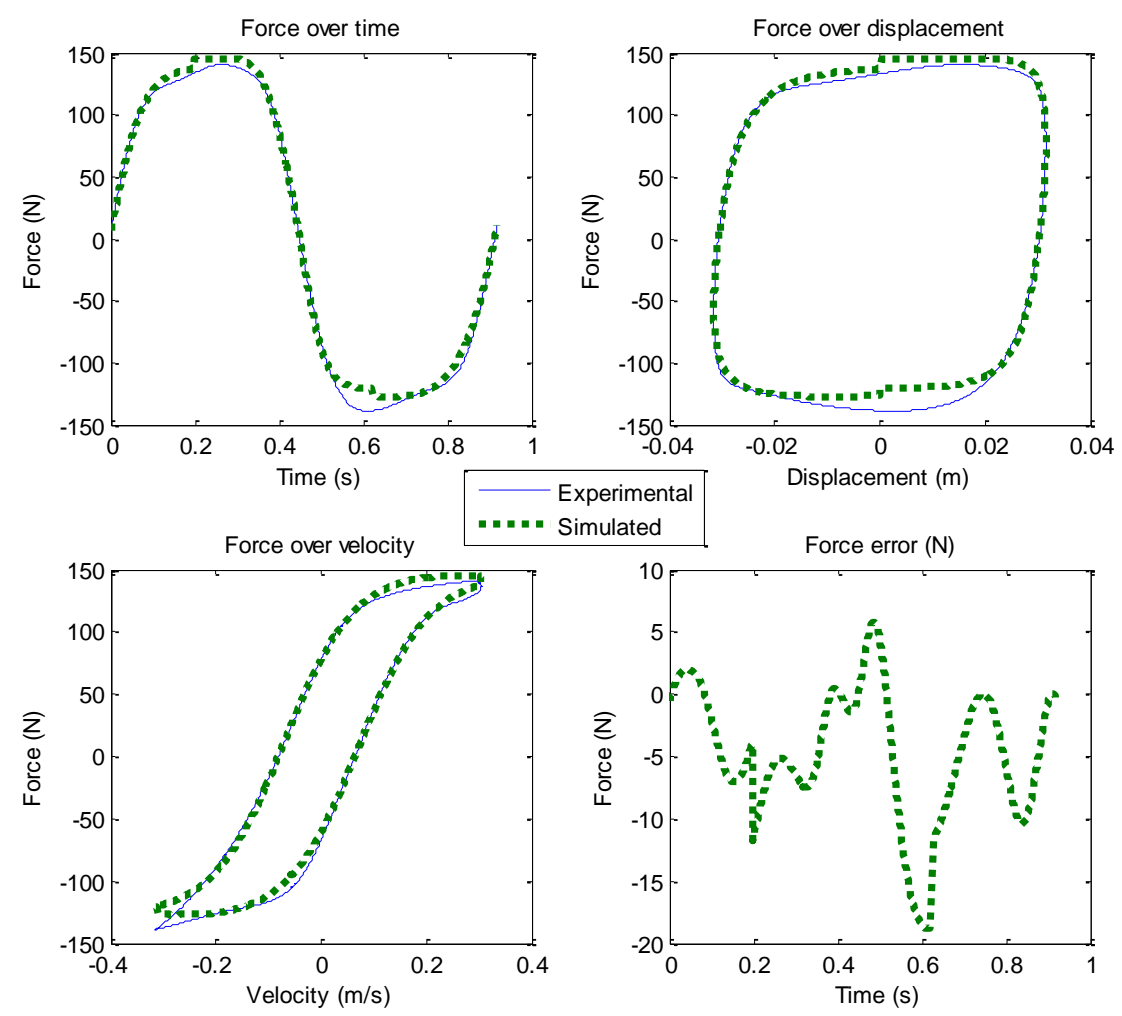

Figure 5. Data comparison of Bouc-Wen/Kwok model for $0.0 \mathrm{~A}$

Table 3. Parameter values for various test conditions of Bouc-Wen/Kwok model

\begin{tabular}{lccccc}
\hline & \multicolumn{5}{c}{ Test current conditions (A) } \\
\cline { 2 - 6 } Parameters & 0 & 0.5 & 1 & 1.5 & 2 \\
\hline Viscosity coefficient, $c$ & -19.03 & 20.07 & 160.75 & 956.04 & 1092.49 \\
$\begin{array}{l}\text { Stiffness coefficient, } k \\
\text { Scaling factor of }\end{array}$ & 25.51 & -321.96 & 4.21 & 1453.66 & 13.55 \\
hysteresis, $\alpha$ & 142.85 & 333.96 & 463.38 & 445.53 & 658.72 \\
$\begin{array}{lcccc}\text { Damper force offset, } f_{0} \\
\text { Hysteresis parameter, } \beta\end{array}$ & 9.10 & 36.71 & 28.93 & -2.60 & -3.36 \\
Hysteresis parameter, $\delta$ & 0.52 & 0.59 & 0.47 & 0.50 & 0.53 \\
\hline
\end{tabular}

\section{CONCLUSIONS}

An alternative approach to the parametric modeling of an MR damper using the PSO method has been presented and successfully applied. By using the experimental test rig data, the non-linear characteristics of the MR damper can be captured without having to resort to its dynamic model (equations of motion). The PSO model responses and the actual test rig outputs are almost identical, which implies that the PSO model has 
captured the real MR damper characteristics. Further rigorous investigation should be carried out to evaluate the proposed model's performance compared with other methods. The results of this study can also be used as a basis to design a more complex semi-active suspension control system involving intelligent methods.

\section{ACKNOWLEDGEMENTS}

The authors would like to be obliged to Universiti Malaysia Pahang for providing laboratory facilities and financial assistance under project no. RDU1303123.

\section{REFERENCES}

[1] Hrovat D. Survey of advanced suspension developments and related optimal control applications. Automatica. 1997;33:1781-817.

[2] Bossis G, Lacis S, Meunier A, Volkova O. Magnetorheological fluids. Journal of Magnetism and Magnetic Materials. 2002;252:224-8.

[3] Ismail I, Mazlan SA, Zamzuri H, Olabi AG. Fluid-particle separation of magnetorheological fluid in squeeze mode. Japanese Journal of Applied Physics. 2012;51:067301.

[4] Ahn KK, Truong DQ, Islam MA. Modeling of a magneto-rheological (MR) fluid damper using a self tuning fuzzy mechanism. Journal of Mechanical Science and Technology. 2009;23:1485-99.

[5] Jaleel JA, Rasheed K. Magneto-Rheological (MR) Damper based Vehicle Suspension. International Journal of Applied Engineering Research. 2012;7.

[6] Razman MA, Priyandoko G, Yusoff AR. Bouc-Wen Model Parameter Identification for a MR Fluid Damper Using Particle Swarm Optimization. Advanced Materials Research. 2014;903:279-84.

[7] Boada M, Calvo J, Boada B, Díaz V. Modeling of a magnetorheological damper by recursive lazy learning. International Journal of Non-Linear Mechanics. 2011;46:479-85.

[8] Dong X-m, Yu M, Liao C-r, Chen W-m. Comparative research on semi-active control strategies for magneto-rheological suspension. Nonlinear Dynamics. 2010;59:433-53.

[9] Wang D, Liao WH. Magnetorheological fluid dampers: a review of parametric modelling. Smart materials and structures. 2011;20:023001.

[10] Dominguez A, Sedaghati R, Stiharu I. A new dynamic hysteresis model for magnetorheological dampers. Smart materials and structures. 2006;15:1179.

[11] Eberhart RC, Shi Y, Kennedy J. Swarm intelligence: Elsevier; 2001.

[12] Eberhart RC, Shi Y. Particle swarm optimization: developments, applications and resources. Evolutionary Computation, 2001 Proceedings of the 2001 Congress on: IEEE; 2001. p. 81-6.

[13] Kwok N, Ha Q, Nguyen T, Li J, Samali B. A novel hysteretic model for magnetorheological fluid dampers and parameter identification using particle swarm optimization. Sensors and Actuators A: Physical. 2006;132:441-51. 